\title{
High Fat Diet Dysbiotic Mechanism of Decreased Gingival Blood Flow
}

\author{
Dragana Stanisic 1,2, Nevena Jeremic ${ }^{2,3 *}$, Suravi Majumder ${ }^{2}$, Sathnur Pushpakumar ${ }^{2}$, \\ Akash George ${ }^{2}$, Mahavir Singh ${ }^{2}$ and Suresh C. Tyagi ${ }^{2}$ \\ ${ }^{1}$ Department of Dentistry, Faculty of Medical Sciences, University of Kragujevac, Kragujevac, Serbia, ${ }^{2}$ Department of \\ Physiology, School of Medicine, University of Louisville, Louisville, KY, United States, ${ }^{3}$ Department of Pharmacy, Faculty of \\ Medical Sciences, University of Kragujevac, Kragujevac, Serbia
}

OPEN ACCESS

Edited by:

Gerald A. Meininger,

University of Missouri,

United States

Reviewed by:

Ahmed A. Elmarakby,

Augusta University,

United States

Jonathan Steven Alexander,

Louisiana State University Health

Shreveport, United States

*Correspondence:

Nevena Jeremic

nbarudzic@hotmail.com

Specialty section:

This article was submitted to

Vascular Physiology,

a section of the journal

Frontiers in Physiology

Received: 03 November 2020 Accepted: 09 February 2021

Published: 03 March 2021

Citation:

Stanisic D, Jeremic N, Majumder S,

Pushpakumar S, George A,

Singh M and Tyagi SC (2021) High

Fat Diet Dysbiotic Mechanism of

Decreased Gingival Blood Flow.

Front. Physiol. 12:625780.

doi: 10.3389/fphys.2021.625780
The gut microbiome has a very important role in human health and its influence on the development of numerous diseases is well known. In this study, we investigated the effect of high fat diet (HFD) on the onset of dysbiosis, gingival blood flow decreases, and the periodontal matrix remodeling. We established a dysbiosis model (HFD group) and probiotic model by Lactobacillus rhamnosus GG (LGG) treatment for 12 weeks. Fecal samples were collected $24 \mathrm{~h}$ before mice sacrificing, while short chain fatty acids (SCFA) analysis, DNA extraction, and sequencing for metagenomic analysis were performed afterwards. After sacrificing the animals, we collected periodontal tissues and conducted comprehensive morphological and genetic analyses. While HFD reduced Bacteroidetes, SCFA, and gingival blood flow, this type of diet increased Firmicutes, lipopolysaccharide (LPS) binding protein, TLR4, pro-inflammatory cytokines (TNF- $\alpha, I L-1 \beta$, and IL-6), matrix metalloproteinases (MMP-2 and MMP-9) expression, and also altered markers of bone resorption (OPG and RANKL). However, LGG treatment mitigated these effects. Thus, it was observed that HFD increased molecular remodeling via inflammation, matrix degradation, and functional remodeling and consequently cause reduced gingival blood flow. All of these changes may lead to the alveolar bone loss and the development of periodontal disease.

Keywords: gut microbiota, lipopolysaccharide, matrix metalloproteinases, Laser Doppler flowmetry, periodontal disease, Lactobacillus rhamnosus, blood flow

\section{INTRODUCTION}

Changes in lifestyle influence usual diet habits and it is already established that consumption of diets with a high content of fats, proteins, and sugars modulate the gut microbiota and consequently affect the whole organism (Tremaroli and Bäckhed, 2012). Gastrointestinal tract presents one of the primary sites for bacterial colonization. More than 1,000 species of bacteria form intestinal flora which is primarily composed of anaerobes that make up more than $99 \%$ of gut microbes including Firmicutes, Bacteroidetes, Proteobacteria, Archaebacteria, and Actinomycetes. More than 90\% of those mentioned are Firmicutes and Bacteroidetes (Zhang and Yang, 2016). The ratio of Firmicutes and Bacteroidetes differs depending on age evolves according to the different age group. For newborns, ratio of 0.4 was measured, 10.9 for adults, and 0.6 for the elderly (Mariat et al., 2009). High fat diet (HFD) leads to a reduction in Bacteroidetes and an 
increase in Firmicutes levels (Murphy et al., 2015). These changes have been implicated in a numerous diseases (Murphy et al., 2015), including periodontitis (Chen et al., 2017; Jia et al., 2019; Yu et al., 2019; Curtis et al., 2020). In addition, HFD increases the number of pathogenic bacteria in the gut, resulting in enhanced lipopolysaccharide (LPS) and lipopolysaccharide binding protein (LBP) production (Hersoug et al., 2018). Furthermore, HFD increases gut permeability, inflammatory response, and oxidative stress. At the same time, this diet decreases the production of short chain fatty acids (SCFA; Lu et al., 2018).

Moreover, HFD upsets the balance in good and bad gut bacteria, LPS production (Potter et al., 2016; Hersoug et al., 2018), decreases SCFA production (Lu et al., 2018), and affects blood vessels and blood flow (Wilde et al., 2000; Bui et al., 2010). Periodontium, gingiva, periodontal ligament, as well as alveolar bone, are greatly vascularized tissues. Adequate blood supply, as well as a healthy vascular endothelial system, is necessary for their function and nutrition (Nguyen et al., 2015; Veronesi et al., 2018; Komaki, 2019). Maintaining the equilibrium between matrix metalloproteinases (MMP) and their inhibitors in the tissues has a crucial role in keeping the endothelial system in good condition (Donnini et al., 2004; Rodrigues and Granger, 2015). Disturbance in local vascular flow is in correlation with inhibition of tissue oxygenation and reduction of microvascular blood flow. Disorder of blood flow activity is noted to be an early marker for progression of microvascular pathology (Clough et al., 2017; Mitra et al., 2018; Ohsugi et al., 2019). HFD contributes to endothelial dysfunction and loss of integrity (Clough et al., 2017; Mitra et al., 2018; Münch et al., 2019) it has been shown that $2 \mathrm{~h}$ after a fatty meal, HFD causes endothelial damage by vascular occlusion and leads to decreased blood flow (Bui et al., 2010; Münch et al., 2019). Reduced blood flow in periodontal tissue, especially in gingiva, can lead to periodontal disease.

According to available literature data, periodontal disease is a dysbiosis condition of the oral microbiome (Payne et al., 2019). Qualitatively and quantitatively altered oral microorganisms induce an enhanced immune response (Gao et al., 2018; Graves et al., 2019). Thus, modified microorganisms can cause systemic inflammation by reaching microorganisms in the gut thorough swallowing and hematogenously dissemination of proinflammatory cytokines [Interleukin-1 Beta (IL-1 $\beta$ ), Interleukin-6 (IL-6), Tumor Necrosis Factor Alpha (TNF- $\alpha)$ ] or bacteria. Gut dysbiosis causes an increase in the gut mucosa permeability resulting in endotoxemia and systemic inflammation (Torres et al., 2017) which consequently causes intensified bone resorption. The production of proinflammatory cytokines, TNF- $\alpha$, IL- $1 \beta$,

\footnotetext{
Abbreviations: ABC, Alveolar bone crest; CEJ, Cement enamel junction; DNA, Deoxyribonucleic acid; ECM, Extracellular matrix; H\&E, Hematoxylin and eosin; HFD, High fat diet; IL-1 $\beta$, Interleukin-1 beta; IL-6, Interleukin-6; LBP, Lipopolysaccharide binding protein; LGG, Lactobacillus rhamnosus GG; LPS, Lipopolysaccharide; MMP, Matrix metalloproteinase; OPG, Osteoprotegerin; PBS, Phosphate-buffered saline; P. gingivalis, Porphyromonas gingivalis; RANKL, Receptor activator of nuclear factor kappa- $B$ ligand; RNA, Ribonucleic acid; RT-PCR, Realtime polymerase chain reaction; SCFA, Short chain fatty acids; TLR4, Toll-like receptor 4; TNF- $\alpha$, Tumor necrosis factor alpha.
}

Interleukin-17 (IL-17) promotes survival, proliferation, osteoclasts activity, and suppression of osteoblasts activity. The cytokines IL-1 $\beta$, TNF- $\alpha$, and IL-17 cause osteoblasts to enhance the receptor activator of nuclear factor kappa- $B$ ligand (RANKL) release. RANKL binds to RANK on osteoclasts precursors and promotes osteoclastogenesis. Increscent in number of osteoclasts leads to resorption of the bone (Hajishengallis, 2015; Torres et al., 2017). HFD also promotes the formation of periodontal disease (Li et al., 2015; Virto et al., 2018a) via an increase in cytokine levels and alveolar bone loss (Fujita and Maki, 2016; Virto et al., 2018a,b). Virto et al. (2018a) indicated an increase in alveolar bone loss by $27.71 \%(2.28 \mu \mathrm{m})$ in the HFD group compared to the control group.

It would be very useful to improve and supplement the diet with probiotics with the aim of obtaining a healthy gut microbiota, prevention, and treatment of gastrointestinal diseases. This will also be beneficial in sense of maintaining gingival blood flow at basal level with consequent prevention of periodontitis development. Lactobacillus rhamnosus GG (LGG) is a Gram-positive rod-shaped facultative anaerobe, capable of surviving low levels of $\mathrm{pH}$ in the stomach and bile acids in the duodenum. It is one of the most used probiotics microorganisms in the world. Studies also proved its beneficial effect in the prevention and treatment of atopic dermatitis (Huang et al., 2017), hypercholesterolemia (Shimizu et al., 2015), and obesity (Sanchez et al., 2014). This probiotic does not ferment sucrose and lactose, so it has been suggested that this anaerobe could significantly reduce the risk of caries (Gruner et al., 2016; Jiang et al., 2018). Moreover, LGG possesses significant in vivo antiinflammatory properties (Lin et al., 2009). However, its effect in the treatment of gingival blood flow and periodontitis has not been fully investigated. Given its non-cariogenic and anti-inflammatory properties, LGG turned out to be a good candidate in the prevention and treatment of periodontal disease (Gatej et al., 2018). The aim of this study was to prove the influence of HFD and LGG on the state of the gut microbiota, gingival blood flow, and the development of periodontal disease (Figure 1).

\section{MATERIALS AND METHODS}

\section{Animal Maintenance and Diet Protocol}

Male wild-type $(\mathrm{C} 57 \mathrm{BL} / 6 \mathrm{~J})$ mice were purchased from the Jackson Laboratory (Bar Harbor, ME). All animals were 8 weeks-old and maintained in 12:12 h light-dark cycle in the animal facility of the University of Louisville. All animal protocols and care were carried out according to the guidelines of the National Institute of Health (NIH) and were approved by the Institutional Animal Care and Use Committee (IACUC \#19592) of the University of Louisville, KY.

Animals were divided into four experimental groups: (1) Wild-type C57BJ/L6 mice (WT), (2) HFD-supplemented wild-type mice (HFD), (3) LGG-supplemented wild-type mice (LGG), and (4) HFD and LGG-supplemented wild-type 


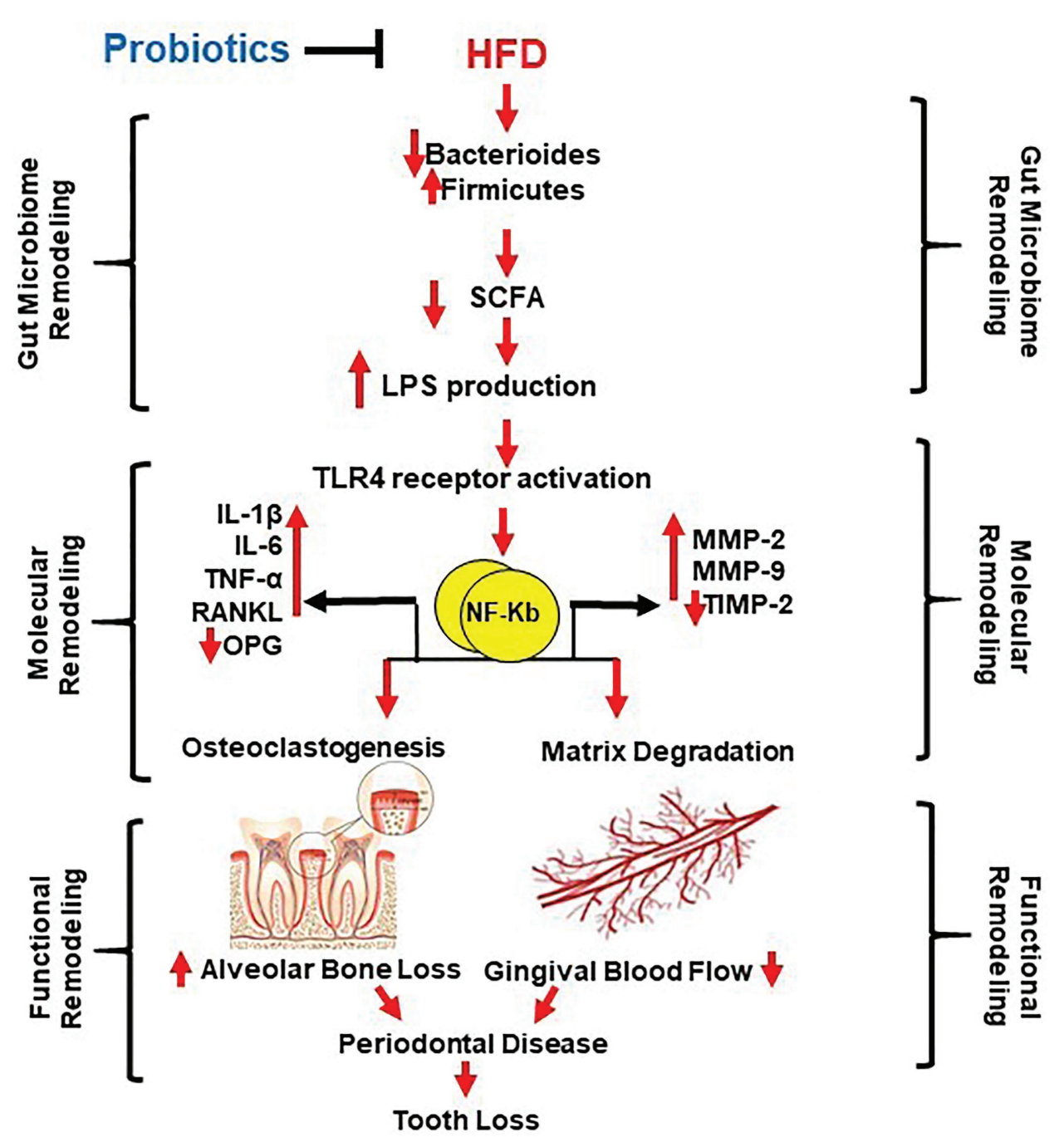

FIGURE 1 I Schematic representation of the study aim. High fat diet (HFD) causes gut dysbiosis (via decreased short chain fatty acids (SCFA) and Bacteroides, and increased Firmicutes and Proteobacteria) resulting in increased lipopolysaccharide (LPS) production and LPS/TLR4 pathway activation in the oral cavity and gastrointestinal tract. Consequently, molecular remodeling occurs by increasing proinflammatory cytokines (IL-1 $\beta$, IL-6, and TNF- $\alpha$ ) and matrix metalloproteinases (MMP-2 and MMP-9). Due to the mentioned consequences, functional remodeling occurs, by an increase of proinflammatory cytokines, RANKL, and the decrease of OPG. Osteoclastogenesis and alveolar bone loss are promoted by disturbance in expression and activity of MMPs, matrix degradation, and gingival blood flow. This together cause periodontal disease and possibility of tooth loss. Probiotics maids mitigate all these consequences.

mice (HFD + LGG; $n=5$ /groups). To create dysbiosis condition, the mice were fed with an HFD (TD.88137; Harlan Laboratories Inc., Indianapolis, IN, United States) for a period of 12 weeks, and with or without oral probiotic LGG@ $9.5 \times 10^{5} \mathrm{CFU}$ for a period of 12 weeks in drinking water (Gatej et al., 2018; He et al., 2018; George et al., 2020). Mice in the control group were fed with the standard chow diet. The standard diet contained 20\% protein, $53 \%$ starch, 9\% fat, and 5\% fiber, while the HFD contained $15.3 \%$ protein, $42.7 \%$ starch, and $42.0 \%$ fat (TD.88137; Harlan Laboratories Inc., Indianapolis, IN, United States). Appropriate diets (standard or HFD, depending on the group) and water are provided ad libitum.

\section{Measurement of Blood Glucose Levels}

Glucose levels in all examined groups were measured according to manufacturer's instructions for commercially available kit (OneTouch Ultra2, LifeScan, Inc).

\section{Gingival Blood Flow Measurement}

Gingival blood flow was measured as it was previously described, in break before procedure animals were under an intraperitoneal combination of anesthetics: ketamine (Ketamine $80 \mathrm{mg} / \mathrm{kg}$ ) and xylazine (Xylazine; $10 \mathrm{mg} / \mathrm{kg}$ ). The gingival blood flow was measured by Laser Doppler using Speckle Contrast Imager (Moor FLPI, Wilmington, DE) at room temperature. The camera 
(580 9752 resolution) was positioned $15 \mathrm{~cm}$ from the buccal surface of the gingiva (Clarke and Shephard, 1984; Baab and Oberg, 1987; George et al., 2018).

\section{Microbiota Analysis}

Fecal samples were collected $24 \mathrm{~h}$ before animal sacrifice and subsequently analyzed at Microbiome Insights Inc.; Vancouver, BC V6R 4K6; Canada. The following analyzes were performed to detect gut dysbiosis: analysis of SCFA, Deoxyribonucleic acid (DNA) extraction and sequencing, and sample preparation according to an already existing database [DNA Extraction + Library preparation and sequencing on the Illumina Next Seq (2× 150 BP); Zhao et al., 2006; Kozich et al., 2013; Bolger et al., 2014; Clarke et al., 2019; Wood et al., 2019].

\section{Measurement of LBP}

Determination of LBP concentration from serum was archived by using ELISA kit with following all already established manufacturer's protocol. We used Mouse LBP ELISA Kit (ab213876, Sigma-Aldrich, St. Louis, Missouri, United States; Chivero et al., 2019).

\section{Gelatin Zymography Analysis}

Activity of MMP was measured using gelatin gel zymography as described previously (Stanisic et al., 2020). For this analysis, we used samples of periodontal tissue and the gels were stained with the Coomassie R-250 brilliant blue dye. The clear digested regions represent MMP activity which were quantified densitometrically using the Un-Scan-It software (Silk Scientific Inc., Orem, UT).

\section{RT-PCR Analysis}

Real-time polymerase chain reaction (RT-PCR) analysis was conducted as it is previously described (Livak and Schmittgen, 2001; George et al., 2018).

We used the right mandibula (periodontal tissue; $n=5$ per group) which were homogenized in a solution of TRIzol reagent (cat no. 15596018; Invitrogen; Thermo Fisher Scientific, Inc., Waltham, MA, United States) The sequences of the primers are shown in Table $\mathbf{1 .}$

\section{Histology and Histomorphometric Analysis}

The mandibular bone and surrounding intact tissues from each euthanized animal were dissected and fixed in $4 \%$ freshly prepared paraformaldehyde (Sigma-Aldrich, St. Louis, MO) from there we used standard procedure which is previously described (Stanisic et al., 2020). In each area of interest, the total number of cells [the fibroblasts were counted manually from images captured at $60 \mathrm{x}$ magnification on Hematoxylin and eosin (H\&E) stained sections]. All quantification and calculations of areas of interest are described previously (Stanisic et al., 2020).

\section{Statistical Analysis}

Data analyses and graphical presentations were performed with GraphPad Prism, version 8 (GraphPad Software, Inc., La Jolla, CA). Data are represented as mean values \pm standard deviation (SD) in five independent experiments in all cases. The experimental groups were compared by one-way analysis of variance (ANOVA) assuming that the values were sampled from Gaussian distributions. For a set of data, if ANOVA indicated a significant difference $(p<0.05)$; Tukey-Kramer multiple comparison tests were used to compare group means. Posttest was only performed if $p<0.05$. If the $p<0.05$ considered statistically significant.

\section{RESULTS}

\section{HFD Effects on the Weight Gain and Blood Glucose Levels}

Values for body weight are shown in Table 2. The significant difference in the values of these parameters was observed between groups in body weights after treatment, while no significant difference was observed in the weight before treatment. A significant increase body weight was observed in the HFD group compared to the control group (Table 2). Moreover, in the group fed with HFD increscent in blood glucose level is obvious compared to control, however, values remain under defined diabetic values and LGG treatment did not statistically affected these levels (Figure 2).

\section{Gut Microbiota Alterations Occur Due to the HFD}

Deoxyribonucleic acid sequencing were done for stool samples from each of the four groups at the end of the experimental protocol to archive an overview of each phylum. We did not

TABLE 1 | Primers used for RT-PCR.

\begin{tabular}{lll}
\hline Target gene & Forward primer sequences (5'-3') & Reverse primer sequences (5'-3') \\
\hline TLR4 & CAA GGG ATA AGA ACG CTG AGA & GCA ATG TCT CTG GCA GGT GTA \\
IL-1 $\beta$ & GAAATGCCATTGACAGTG & CTGGATGCTCTCACTAGGACA \\
IL-6 & CTGCAAGAGACTTCCATCCA & CAGGTCTGTTGGGAGTGGT \\
TNF- $\alpha$ & CATCTTCTCAAATCGAGTGACAA & TGGGAGTAGACAAGGTACAACCC \\
MMP-2 & CTGATGGCACCCATTACACCT & GATCTGAGCGATGCCATCAAA \\
MMP-9 & AGAGATGCGTGGAGAGTCGAA & AAGGTTGGAATCTGCCCAGG \\
TIMP-2 & CAAGTCTCGCCTGCATCAA & TCGAACCCTTGGAGGCT \\
RANKL & CAGCATCGCTCTGTTCCTGTA & CTGCGTTTCATGGAGTCTCA \\
OPG & ACCCAGAACTGGTCATCAGC & CTGCAATACACACACTCATCACT \\
GAPDH & AGGTCGGTGTGAACGGTTTG & TGTAGACCATGTAGTTGAGGTCA
\end{tabular}


TABLE 2 | Body weight measurements, before and after treatment, presented as mean \pm SD.

\begin{tabular}{|c|c|c|c|c|}
\hline Group & WT & HFD & LGG & HFD + LGG \\
\hline BW before (g) & $20.17 \pm 0.5$ & $21.27 \pm 0.83$ & $19.93 \pm 0.72$ & $21.43 \pm 0.47$ \\
\hline BW after (g) & $32.93 \pm 0.4^{*}$ & $39.33 \pm 0.86^{* \#}$ & $34.97 \pm 0.15^{*}$ & $36.9 \pm 0.36^{*}$ \\
\hline
\end{tabular}

BW before vs. BW after treatment " $p<0.05 ; W T$ after vs. HFD after ${ }^{*} p<0.05 ; n=5$.

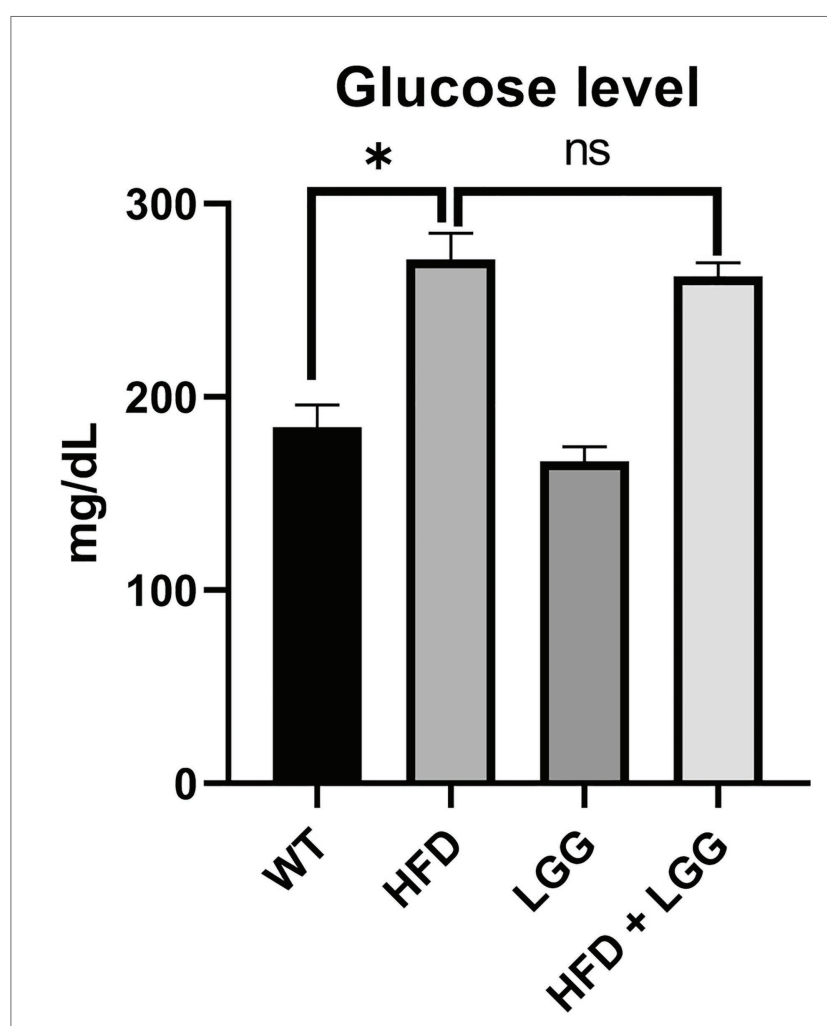

FIGURE 2 | Effect of HFD and Lactobacillus rhamnosus GG (LGG) treatment on the glucose level (ng/ml). WT, Wild-type C57BJ/L6 mice; HFD, HFDsupplemented wild-type mice; LGG, LGG-supplemented wild-type mice; HFD + LGG, HFD, and LGG-supplemented wild-type mice. Values remain under defined diabetic values. All data represent means $\pm S D$, WT vs. HFD group $* 0<0.05$; HFD vs. HFD + LGG ${ }^{n s} p>0.05, n=5$.

find a significant shift in the relative abundance of Firmicutes or Bacteroidetes in the control (relative abundance of Firmicutes was $19.62 \%$ and of Bacteroidetes was 24.73\%; Figures 3A,B). However, the abundance of Firmicutes was increased in the HFD group (42.52\%) with an accompanying decrease in Bacteroidetes abundance (21.74\%; Figures 3A,B). Over and above, LGG treatment alone reduced the number of Firmicutes and increased the number of Bacteroidetes with especially affected HFD + LGG group where the ratio of these bacteria was almost normalized.

The results of our study showed a significant reduction in acetic (Figure 3C), propionic (Figure 3D), butyric (Figure 3E), valeric (Figure 3F), and isovaleric acids (Figure 3G) in stool samples in the HFD group (Figures 3C-G) compared to the control group. Also, a statistically significant increase in propionic, butyric, valeric, and isovaleric acids was observed in the groups treated with LGG compared to the control and HFD groups (Figures 3D-G), while values of acetic acid were not changed (Figure 3C).

\section{HFD Contributes Endotoxemia}

Because of an HFD, there is an increased production of LPS by oral and gut bacteria. The spread of LPS through the bloodstream is made possible by LBP. Levels of LBP were measured in mice in all experimental groups. Compared to the control group, the data showed that the level of LBP significantly increased in the HFD group $(p<0.05)$. Also, data showed that the level of LBP significantly decreased in HFD + LGG group $(p<0.05)$ as compared to HFD mice (Figure 4). These results suggest that HFD, due to changes in the microbiota, affects the increased production of LPS and its spread throughout the whole body.

\section{HFD Causes Expression and Activity Changes in Several Inflammation Pathways in Periodontal Tissues}

We measured the mRNA expression of TNF $\alpha$, IL-1 $\beta$, IL-6, TLR4, MMP-2, MMP-9, TIMP-2, RANKL, and osteoprotegerin (OPG). TNF- $\alpha$, IL- $1 \beta$, and IL- 6 are the most important inflammation markers of periodontal disease, and its increase has been very well explained in periodontitis (Yucel et al., 2015) as a response to infection with the periodontal pathogens (Porphyromonas gingivalis, etc.). MMP-2, MMP-9, and TIMP-2 are responsible for extracellular matrix (ECM) degradation in periodontal tissue (Stanisic et al., 2019, 2020). RANKL and OPG are one of the key indicators of periodontal disease. OPG is osteoclastogenesis inhibitory factor and RANKL is an apoptosis regulator gene, a binding partner of OPG, and osteoclast differentiation factor. The data showed that TNF- $\alpha$, IL-1 $\beta$, IL-6, TLR4, MMP-2, MMP-9, and RANKL all peaked in periodontal tissue in the HFD group of mice. The LGG significantly decreased the expression of TNF- $\alpha$, IL-1 $\beta$, IL- 6 , TLR4, MMP-2, and MMP-9, indicating that LGG treatment attenuated the inflammation of periodontal tissue (Figures 5A-F). LGG did not affect RANKL expression (Figure 5H). OPG and TIMP-2 mRNA expression levels were significantly less in the HFD mice than WT mice (Figures 5G,I).

\section{HFD Contributes Matrix Degradation in Periodontal Tissue}

In our study, we demonstrated a significant increase in the MMP-9 activity in HFD mice in the periodontal tissue samples (Figures 6A,B). This increased MMP-9 activity provoked the 
A

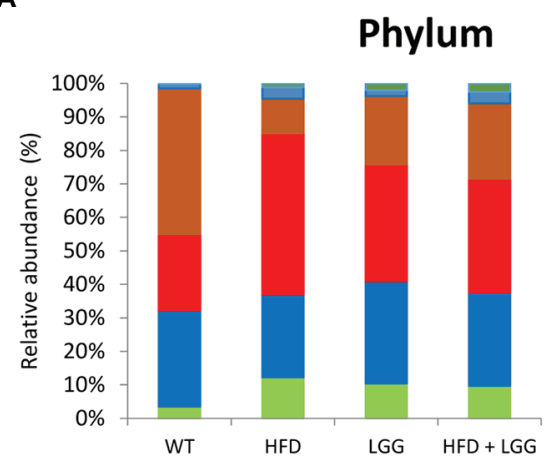

B

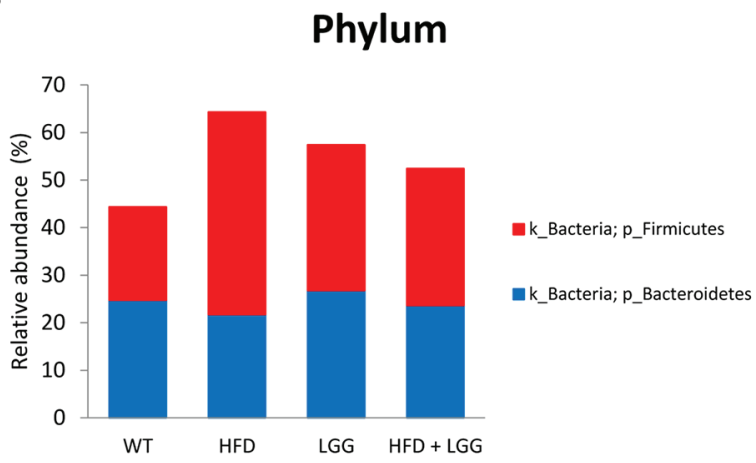

C

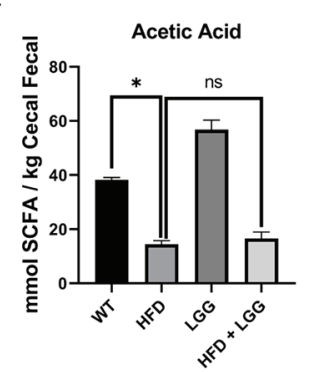

D

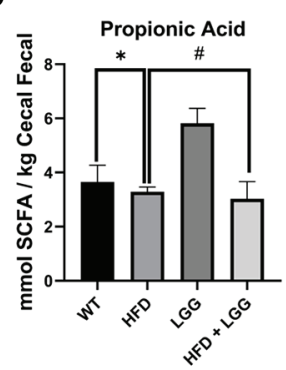

E

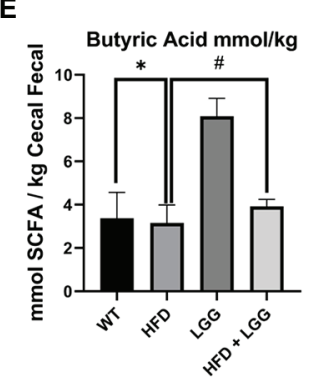

$F$

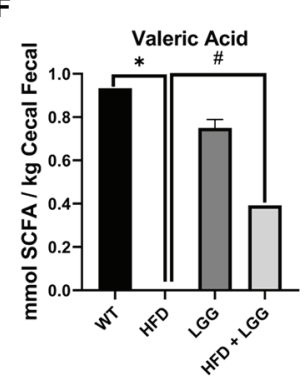

G

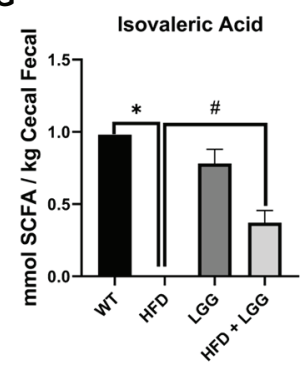

FIGURE 3 | Microbiota analysis of stool in all examined groups. WT, Wild-type C57BJ/L6 mice; HFD, HFD-supplemented wild-type mice; LGG, LGG-supplemented wild-type mice; HFD + LGG, HFD and LGG-supplemented wild-type mice. (A) Relative abundance of total bacteria at the phylum level in each treatment group (\%); (B) Relative abundance of Bacteroides and Firmicutes at the phylum level in each treatment group (\%); (C) Analysis of acetic acid; (D) Analysis of propionic acid; (E) Analysis of butyrate acid; (F) Analysis of valeric acid; (G) Analysis of isovaleric acid. All data represent means \pm SD, WT vs. HFD group * $p<0.05$; HFD vs. HFD + LGG ${ }^{n s} p>0.05$, HFD vs. HFD + LGG ${ }^{*} p<0.05, n=5$.

degradation of matrix proteins resulting endothelial disorder of blood vessels and at the same time a disorder at the level of the periodontium, gingiva, and the alveolar bone. MMPs helped the degradation of the arterial matrix and caused decreased gingival blood flow (Figures 7A,B). The activity of MMP-9 was found to be significantly increased in the HFD mice compared to the WT and HFD + LGG groups of mice (Figure 6B).

\section{HFD Reduces Gingival Blood Flow}

Gingival blood flow is required for conservation of periodontal tissue and tooth function. In the current study, we measured gingival blood flow (Figure 7A). Our results indicate that HFD significantly reduced blood flow $(215.2 \pm 10.9)$ in the gingiva compared to control mice (264.6 \pm 9.5 ; Table 3$)$. Interestingly, LGG treatment alone significantly recover gingival blood flow to basal levels which indicated the same trend in the HFD + LGG group of mice $(247.7 \pm 9.1)$, suggesting that LGG improves gingival blood flow in experimental mice treated with HFD.

\section{HFD Promotes the Development of Periodontal Disease via Losing Alveolar Bone, Loss of Epithelial Downgrowth, and Reducing the Number of Fibroblasts}

Periodontal tissue was stained and observed under a lens with 60x $(200 \mu \mathrm{m})$ to analyze the distance between cement enamel junction to the alveolar bone crest (CEJ-ABC), epithelial downgrowth (ED), and fibroblasts. ED was measured by the distance of the apical migration of epithelial attachment relative to the CEJ (Figure 8A). H\&E staining with histomorphometric analysis showed a smaller number of fibroblasts, an increase in $\mathrm{ED}$, and increased distance between CEJ-ABC in the HFD group (Figures 8B,C). Results in the HFD group showed ED, and alveolar bone-loss compared to the control group (Figures 8B,C). LGG treatment improved all the above parameters (Figures 8B,C). Our results showed a significant reduction in the number of fibroblasts in the HFD group compared to the control group (Figure 8D), which indicates destruction of periodontal tissue and promotes the development of periodontal disease.

\section{DISCUSSION}

Gut microbiota officiates important functions in human physiology and its composition is dependent on environmental factors, including personal hygiene, diet, drug use, and presence of toxins, as well as genetic factors (Turnbaugh et al., 2007; Lozupone et al., 2012; Jandhyala et al., 2015; Semenkovich et al., 2015; Bai et al., 2016). Alteration of the gut microbiome has been implicated in a number of diseases, including periodontitis (Greiner and Backhed, 2011; Komazaki et al., 2017; De Luca and Shoenfeld, 2019; Jia et al., 2019; Curtis et al., 2020). 


\section{Lipopolysaccharide binding protein concentration}

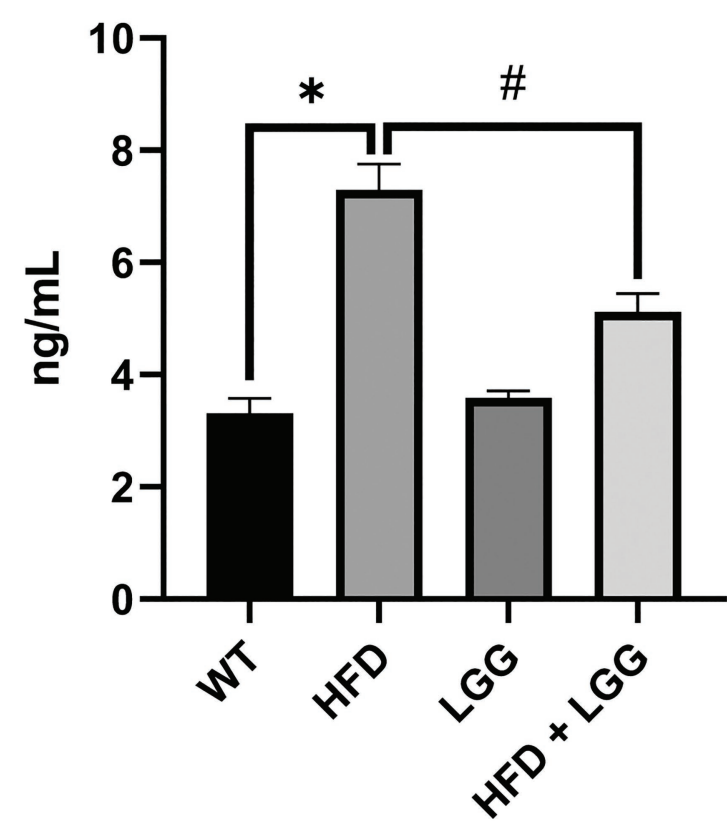

FIGURE 4 | Effect of HFD and LGG treatment on the lipopolysaccharide binding protein concentration in mice serum (ng/ml). WT, Wild-type C57BJ/L6 mice; HFD, HFD-supplemented wild-type mice; LGG, LGG-supplemented wild-type mice; HFD + LGG, HFD, and LGG-supplemented wild-type mice. All data represent means $\pm \mathrm{SD}$, WT vs. HFD group $* 0<0.05$; HFD vs. HFD + LGG ${ }^{*} p<0.05, n=5$.

Bacteroidetes and Firmicutes represent two of the most important phyla which are involved in the gut microbiome. However, a shift in the proportion of bacteria Bacteroidetes and Firmicutes are associated with periodontitis (Chen et al., 2017; Yu et al., 2019). The pivotal role in the composition of the gut microbiota has diet (Scott et al., 2013). After consumption of specific dietary component, the human gut flora composition may significantly change within just a couple of days ( $\mathrm{Ng}$ et al., 2014). Besides increscent in body weight (Table 2), long exposition to HFD has also been shown to ameliorate gut physiology, leading to changes in the composition of gut microbiota, specifically influenced by overexpression of Firmicutes (Turnbaugh et al., 2008). Results of our study are in correlation with previously mentioned (Figures $\mathbf{3 A}, \mathbf{B}$ ). We found that HFD significantly increased the total number of Firmicutes, but with remarkable decrease of Bacteroidetes (Figures 3A,B). On the other hand, LGG supplementation significantly reduced the overall abundance of Firmicutes while substantially increased Bacteroidetes (Figures 3A,B). Moreover, primates have more similar gut microbiota to human and studies of Becker et al. (2019a) on an African green monkey showed increased numbers of Prevotellaceae and Bacteroidetes-Prevotella-Porphiromonas in the established new model of Crohn's disease. Furthermore, they investigated mutual changes in the structure of the intestine and suggested the influence of Firmicutes, Bacteroidetes, Proteobacteria, Archaebacteria, and Actinomycetes on cytokines and its mediators (Becker et al., 2019b). Those observations were in consistence with the results of our study (Figures 3A,B). It still remains unclear how this enrichment influences periodontitis state, making this topic at the high range of interest in scientific world. Taking everything above into consideration, we can possibly say that even small classes and bacteria can have an impact on microbiome in such an essential way that those effects will not be just reflected at the phyla level.

The change in the microbiota composition gives different amounts of metabolites that are produced in the gut (Yang et al., 2019). SCFA are one of the major categories of metabolites produced in the gut. Gut microbiota produces the following SCFA, butyrate, acetate, propionate, valeric, and isovaleric acid (Lu et al., 2014). SCFA play significant roles in many diseases and metabolic disorders such as diabetes (Tolhurst et al., 2012) and obesity-related inflammation (Al-Lahham et al., 2011). Moreover, fermentation of anaerobic bacteria can lead to production of SCFA and might establish themselves in places which are associated with pathology such as periodontal disease (Niederman et al., 1997; Tsuda et al., 2010). The results of our study had shown a significant reduction in acetic, propionic, butyric, valeric, and isovaleric acids in fecal samples in the HFD group compared to the control group. Also, a significant increase in propionic, butyric, valeric, and isovaleric acids was observed in the groups treated with LGG compared to the control and HFD groups (Figures 3D-G), while acetic acid was not significant altered (Figure 3C). This observation suggests that, in our case, HFD through reduction of SCFA can support the reduction of gingival blood flow and the occurrence of periodontal disease (Figures 1, 7; Table 3). Furthermore, in gingival crevicular fluid of patients with chronic periodontitis low levels of propionic and butyric acids were found (Niederman et al., 1997; Qiqiang et al., 2012), and this can prevent tightbinding potential of epithelial cells resulting in penetration of bacterias and destruction of periodontal tissue (Takigawa et al., 2008). Periodontopathogen bacteria is responsible for the production of butyric acid which is mainly involved in the development of periodontal disease onset (Cueno and Ochiai, 2018). Interestingly, direct application of butyric acid in gingival tissue causes activation of NF- $\mathrm{BB}$ in the blood only after 60 to $180 \mathrm{~min}$ (Cueno et al., 2016), indicating its short-term immediate effect. Also, Cueno and Ochiai (2018) studies indicate that elevated values of butyric acid in the gingiva caused the quantity of representative inflammatory markers (Caspases 12 and $1, \mathrm{IL}-1 \beta$, and TNF- $\alpha$ ) to decrease. The results of our study prove precisely that SCFA (Figures 3C-G), reduction increases inflammatory markers such as IL- $1 \beta$ and TNF- $\alpha$ in periodontal tissue (Figures 5B,C). However, the effect of gut butyric acid and periodontal disease has not been sufficiently elucidated and investigated. The reduction of SCFA in the stool affected gut permeability and consequently helped to change the gingival blood flow and the occurrence of inflammation of periodontal tissue. Additional research on the effects of individual action of gut SCFA and periodontal disease 
A

TLR4 Gene Expression
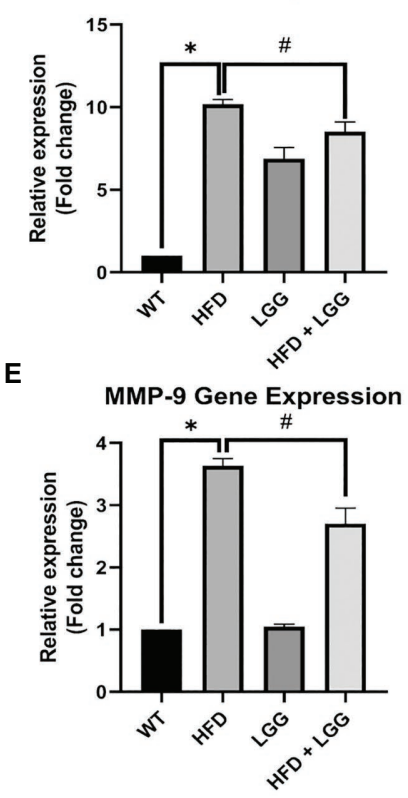

H

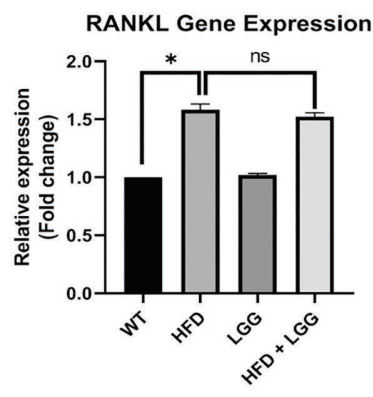

B

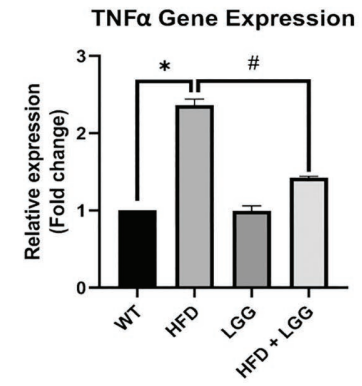

$\mathbf{F}$

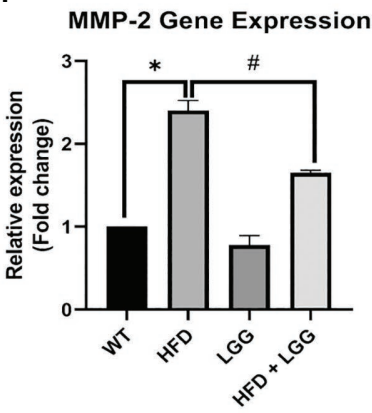

I

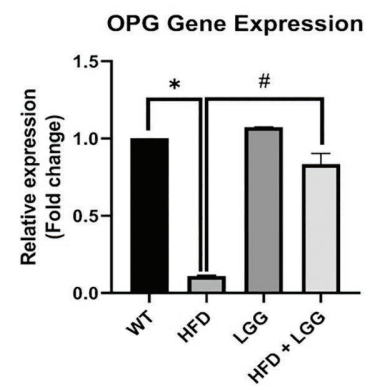

C

IL-1 $\beta$ Gene Expression
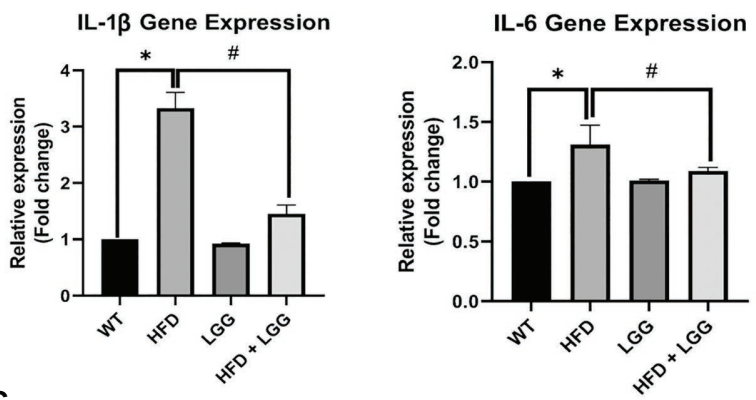

6

TIMP-2 Gene Expression

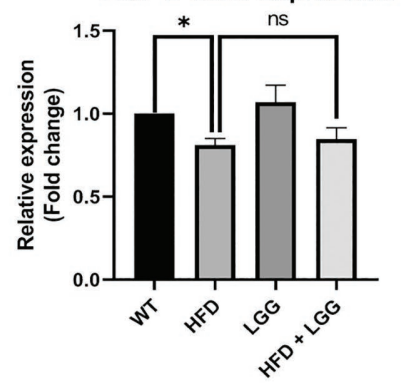

FIGURE 5 | Effect of HFD and LGG treatment on the molecular remodeling in periodontal tissues. WT, Wild-type C57BJ/L6 mice; HFD, HFD-supplemented wildtype mice; LGG, LGG-supplemented wild-type mice; HFD + LGG, HFD, and LGG-supplemented wild-type mice. Quantitative RT-PCR analysis for (A) TLR ${ }_{4}$,

(B) TNF $\alpha$, (C) IL-1 individual mRNAs with normalization GAPDH, using the $2^{-\triangle \Delta C a}$ method. All data represent means $\pm S D$, Fold change vs. WT group. All data represent means \pm SD, WT vs. HFD group " $p<0.05$; HFD vs. HFD + LGG; ns $p>0.05$, HFD vs. HFD + LGG ${ }^{*} p<0.05, n=5$.

is needed to elucidate the mechanisms of their action. In summary, we can observe that HFD can support the reduction of gingival blood flow and the occurrence of periodontal disease through reduction of SCFA.

Live bacterias and LPS as bacterial component, can change location from the gut lumen to other tissue sites (Scheithauer et al., 2016). LPS, as the most important component of the Gram-negative bacteria membrane, binds to Toll-like receptor 4 (TLR4) and activates innate immune response. In our study, elevated level of this receptor expression was observed in the HFD group (Figure 5A; Wei et al., 2020). LPS transfer to the TLR4 is catalyzed by local and systemically LPS binding protein. Furthermore, hepatocytes are responsible for the production of LBP which blood levels are increased in chronic and aggressive periodontitis (Soolari et al., 1999; Wohlfeil et al., 2012). We measured the level of LBP in the mice serum in order to detect another potential route of periodontal disease (HFD gut
dysbiosis-LPS/TLR4 pathway-gingival blood flow-periodontal disease). The results of our study showed that LBP was significantly increased in HFD group compared to the control group, while in the HFD + LGG group a decrease of LBP levels compared to the HFD group was observed (Figure 4).

According to a recent study, we also have investigated the role of HFD during inflammation and its possibility to cause periodontal tissue damage (Muluke et al., 2016). The expression of TNF $\alpha$, IL-1 $\beta$, IL-6, TLR4, RANKL, and OPG in periodontal tissue of mice was measured. HFD promotes the development of gingivitis and periodontitis by systemic and local inflammatory response. Blasco-Baque et al. (2012) showed that TNF- $\alpha$, IL-1 $\beta$, and IL-6 periodontal tissue expression were increased in the periodontal tissue of HFD mice. It has been shown that inflammatory cytokines such as TNF- $\alpha$, IL-1 $\beta$, IL-6 are in tight correlation with development of periodontal disease (Pacios et al., 2012; Cekici et al., 2014). Jin et al. (2013) showed 
A

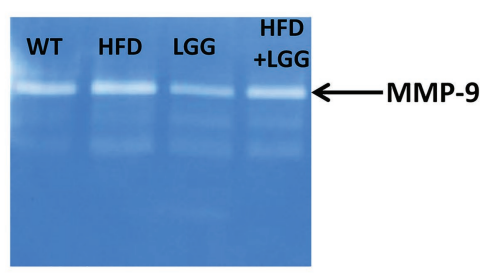

B

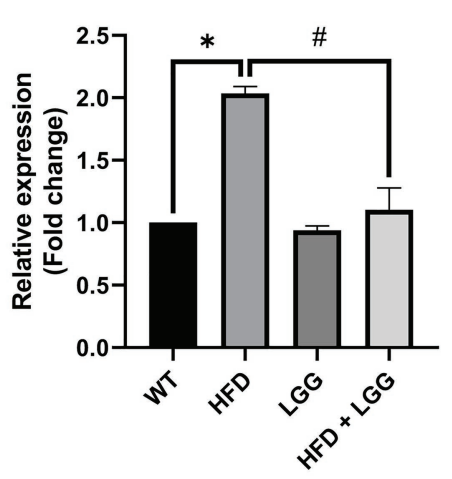

FIGURE 6 | Effect of HFD and LGG treatment on the MMP activity in periodontal tissues. WT, Wild-type C57BJ/L6 mice; HFD, HFD-supplemented wild-type mice; LGG, LGG-supplemented wild-type mice; HFD + LGG, HFD and LGG-supplemented wild-type mice. (A) Original zymography membrane. (B) Gelatin zymography analysis in periodontal tissue. All data represent means $\pm \mathrm{SD}$, WT vs. HFD group " $p<0.05$; HFD vs. HFD $+\mathrm{LGG} " p<0.05, n=5$.

A

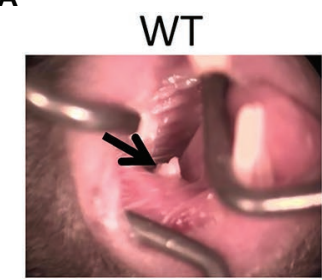

B

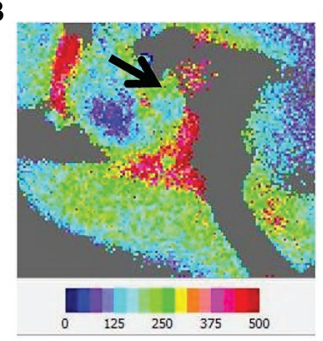

HFD
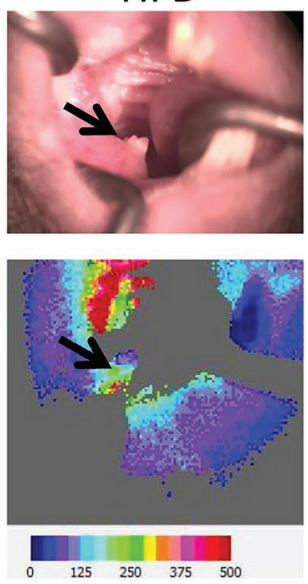

LGG
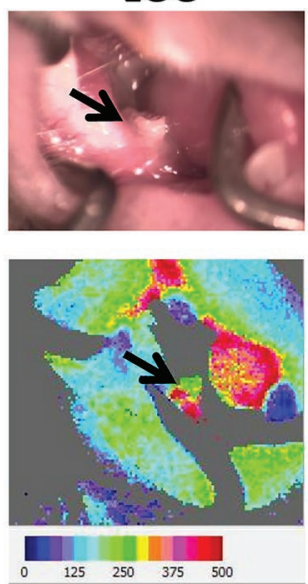

$H F D+L G G$
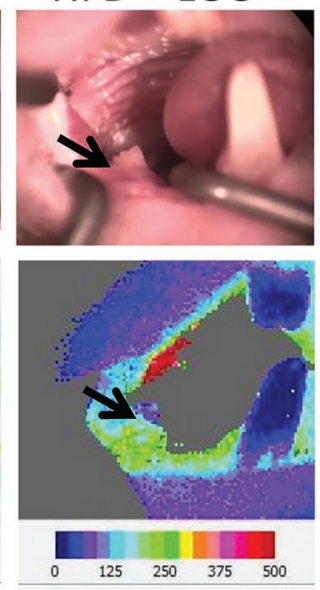

FIGURE 7 | Effect of HFD and LGG treatment on the gingival blood flow. WT, Wild-type C57BJ/L6 mice; HFD, HFD-supplemented wild-type mice; LGG, LGGsupplemented wild-type mice; HFD + LGG, HFD, and LGG-supplemented wild-type mice. (A) Buccal view of the exposed right mandibular gingiva in first molar area. (B) Blood flow imaging at the surface of the buccal right mandibular gingiva in first molar area via Doppler laser. The black arrows indicate the gingival blood flow measurement site.

TABLE 3 | Gingival blood flow measurements presented as mean \pm SD.

\begin{tabular}{lcccc}
\hline Group & WT & HFD & LGG & HFD + LGG \\
\hline $\begin{array}{l}\text { Flux statistic } \\
(\mathrm{PU})\end{array}$ & $264.6 \pm 9.5$ & $215.2 \pm 10.9^{*}$ & $247.5 \pm 8.3$ & $247.7 \pm 9.1^{\#}$ \\
\end{tabular}

WT vs. HFD group * $p<0.05 ;$ HFD vs. HFD + LGG $p<0.05 ; n=5$.

LPS/TLR4 pathway might have a pivotal role when it comes to exposure of macrophages to LPS due to palmitic acid which may provoke increased production of inflammatory cytokines specifically through this pathway. Our study of periodontal tissue overlaying areas of alveolar bony defects revealed that HFD animals indeed exhibited higher expression of TNF- $\alpha$,
TNF $\alpha$, IL-1 $\beta$, IL-6, TLR4, and RANKL (Figures 5A-D,H) and lower expression of OPG (Figure 5I) compared with control animals, suggesting that HFD promotes local inflammatory activation in response to infection.

Elevated proinflammatory cytokines production induces increased activity and expression of MMP. Previously conducted experimental studies have found that HFD causes loss of vascular elasticity, primarily due to deposition of harmful substances in the walls of blood vessels, which in turn leads to reduction of blood flow (Declèves et al., 2013; Mitra et al., 2018; Yuan et al., 2019). Since MMPs are one of the factors causing the occlusion as well as damage of blood vessels and periodontal tissue, MMP-2, MMP-9, and TIMP-2 expression was measured in periodontal mice tissue. MMPs are responsible for ECM degradation catalyzed 


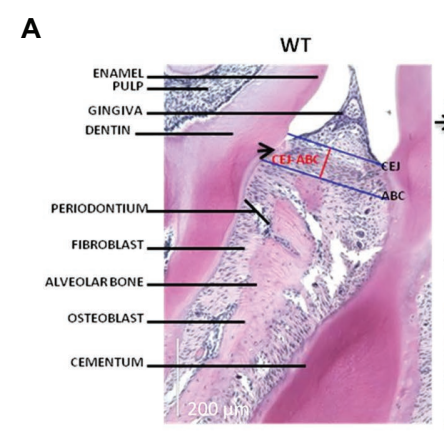

B

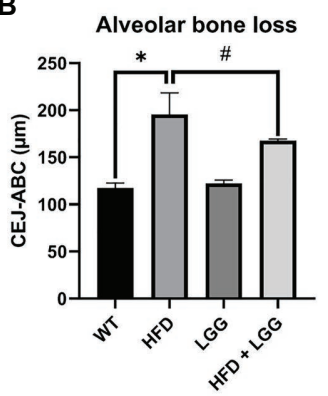

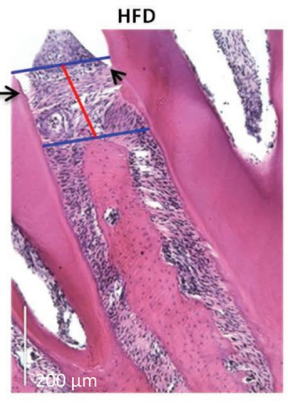

C

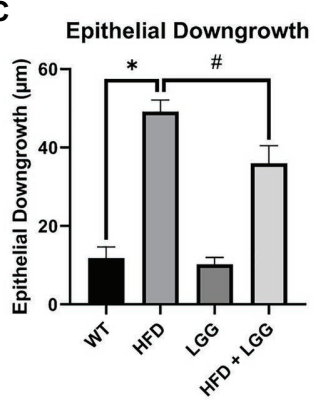

LGG
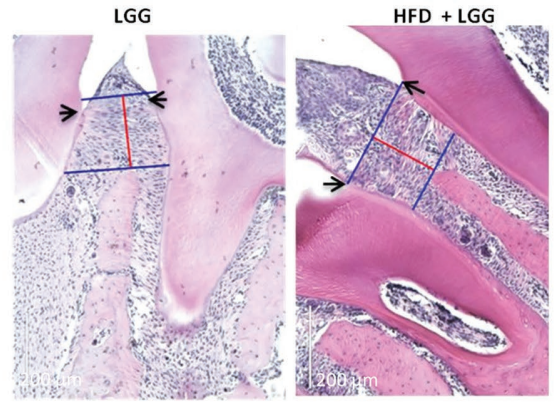

D

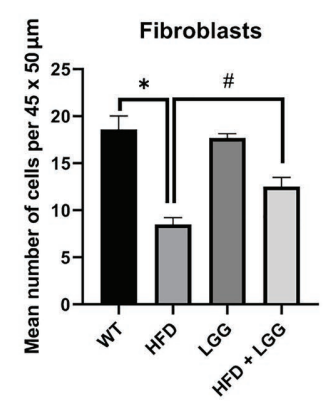

FIGURE 8 | Representative images of hematoxylin-eosin (H\&E) staining of periodontal tissue and histomorphometric analysis of alveolar bone loss, ED, and number of fibroblasts per $45 \times 50 \mu \mathrm{m}$. WT, Wild-type C57BJ/L6 mice; HFD, HFD-supplemented wild-type mice; LGG, LGG-supplemented wild-type mice; HFD + LGG, HFD, and LGG-supplemented wild-type mice. (A) Representative depiction of histological sections. Images were captured at $20 \times(200 \mu \mathrm{m})$ magnification after H\&E staining. Blue lines represent alveolar bone crest (ABC), and cement enamel junction (CEJ). Red lines represent the alveolar bone loss. Black arrows show the apical extent of the epithelial downgrowth (ED). (B) Alveolar bone-loss was measured as the distance between the CEJ and the ABC. (C) ED was defined by measuring the distance from the cementoenamel junction (CEJ) to the apical extent of the junctional epithelium. Results are expressed in $\mu \mathrm{m}$. (D) Bars shows mean number of fibroblasts per $45 \times 50 \mu \mathrm{m}$. Scale bars $=200 \mu \mathrm{m}$. All data represent means $\pm \mathrm{SD}$, WT vs. HFD group $" p<0.05 ;$ HFD vs. HFD + LGG ${ }^{*} p<0.05, n=5$.

by zinc-dependent endopeptidases. MMPs can selectively digest pathological ECMs in different models of disease or injury. Additionally, improper digestion of ECMs can lead to fibrosis in different tissues (Chen and $\mathrm{Li}, 2009$ ). The results of this study showed that MMP-2 and MMP-9 were significantly increased in the HFD group, while the HFD + LGG group reduced mRNA levels from MMPs (MMP-2 and MMP-9) compared to HFD groups (Figures 5E,F). Moreover, it was noticed that the expression and activity of MMP-9 were increased in experimental periodontitis, regardless of the method of periodontitis induction (Barreiros et al., 2018). These studies indicated the importance of ECM degradation as one of the factors in the periodontal disease development. In our study, MMP-9 activity was measured by gelatin zymography in periodontal mice tissue. The results showed that MMP-9 activity was significantly increased in the HFD group, while the LGG treatment reduced the level of MMP-9 activity compared to the HFD group (Figure 6A).

Previously conducted study had shown that HFD causes endothelial damage after $2 \mathrm{~h}$ of meal via vascular occlusion, leading to a reduction in blood flow, which is consistent with our results (Bui et al., 2010; Münch et al., 2019). We used HFD to determine its effect on periodontal tissues, which can be direct on the oral cavity, or indirect on the blood vessels, blood flow, and gastrointestinal tract (Figure 1). Blood flow in the gingiva indicates circulation in the gingival and periodontal tissue and their nutrition and function. We measured gingival blood flow by using Laser Doppler. In the buccal gingiva region of the first right mandibular molar, GBF was $215.2 \pm 10.9$ flux units in the HFD group, while in the control group it was $264.6 \pm 9.5$ flux units (Table 3). Because of HFD, there was a reduction in gingival blood flow while LGG in the HDF + LGG group improved gingival blood flow $(247.5 \pm 9.1$ flux unit). For the first time in our study, it has been shown the influence of HFD on gingival blood flow in mice (Figures 7A,B). Baab and Oberg (1987) suggested that some significant morphological and changes in vascularization occurred as an early sign of gingivitis in dogs while measured blood flow in the inflamed gingiva did not show very convincing evidence. Furthermore, in previous experimental studies, it was hypothesized that there are three predisposing factors for gingivitis occurrence, including vascular stasis, stress, and smoking. These studies also found a reduction in the gingival blood flow during ischemia in rabbits infused with epinephrine and nicotine (Clarke et al., 1981; Clarke and Shephard, 1984).

Numerous disorders lie in the pathology of periodontal disease development. Firstly, HFD causes periodontal disease (Blasco-Baque et al., 2012), which was also confirmed in our study (Figures 8A-D). We used histomorphometric analysis as reported in previous studies (Semenoff et al., 2008). ED and CEJ-BC were chosen thus their previous correlation with attachment loss and bone-loss (Susin and Rösing, 2003; Breivik et al., 2006). Histomorphometric analysis revealed an 
increase in the distance of $\mathrm{CEJ}-\mathrm{ABC}$ in the HFD group, which indicates the existence of periodontal disease (Figure 8B). For histomorphometric analysis, the mean distance from the $\mathrm{CEJ}-\mathrm{ABC}$ and the mean $\mathrm{ED}$ in $\mu \mathrm{m}$ was statistically significantly higher in the HFD mice group ( $p<0.05$ vs. WT; Figures 8A-C). Based on studies of similar design and obtaining the values for the distance $\mathrm{CEJ}-\mathrm{ABC}$, we can conclude that periodontal disease exists in our mice. In our study, we discovered the difference in periodontium between these four groups of mice, and the influence of HFD and dysbiosis on the periodontium, as well as the occurrence of periodontal disease. HFD mice showed an increase in the periodontal breakdown by molecular remodeling (Figures 5A-I), matrix degradation (Figures 6A,B), decreased gingival blood flow (Table 3), alveolar bone loss (Figure 8B), and decreased number of fibroblasts (Figure 8D). These findings as well are in correlation with previously described. Decreased fibroblast levels were observed in the experimental model of periodontitis in mice compared to healthy controls from wild type group (Alshammari and Amar, 2019).

Our hypothesis was established on the effects of HFD in sense of inducing chronic inflammation throughout increscent of TNF- $\alpha$, IL- $1 \beta$, and IL- 6 levels and on such a way initiating destruction of the periodontium thus reduced the number of fibroblasts. In addition to the above, increased expression of organic acids might be interpreted as the important factor for suppressing nuclear factor kappa- $B$ and inflammation overall. On the other hand, HFD, by suppressing this pathway leads to more intense inflammation and periodontal disease.

The results of this study indicate for the first time the influence of HFD and dysbiosis on gingival blood flow. Also, the results of this study suggest that oral LGG therapy may regulate gingival blood flow. With this study, we provided theoretical evidence for the dysbiotic mechanism of decreased

\section{REFERENCES}

Al-Lahham, S., Roelofsen, H., Rezaee, F., Weening, D., Hoek, A., Vonk, R., et al. (2011). Propionic acid affects immune status and metabolism in adipose tissue from overweight subjects. Eur. J. Clin. Investig. 42, 357-364. doi: $10.1111 / \mathrm{j} .1365-2362.2011 .02590 . \mathrm{x}$

Alshammari, A., and Amar, S. (2019). Proposal for a novel murine model of human periodontitis using Porphyromonas gingivalis and type II collagen antibody injections. Saudi Dent. J. 31, 181-187. doi: 10.1016/j. sdentj.2019.02.043

Baab, D. A., and Oberg, P. A. (1987). Laser Doppler measurement of gingival blood flow in dogs with increasing and decreasing inflammation. Arch. Oral Biol. 32, 551-555. doi: 10.1016/0003-9969(87)90063-X

Bai, Y., Wei, Y., Wu, L., Wei, J., Wang, X., and Bai, Y. (2016). C/EBP $\beta$ mediates endoplasmic reticulum stress regulated inflammatory response and extracellular matrix degradation in LPS-stimulated human periodontal ligament cells. Int. J. Mol. Sci. 17:385. doi: 10.3390/ijms17030385

Barreiros, D., Filho, N. P., Paula-Silva, F. W. G., Oliveira, K. M. H., Lucisano, M. P., Rossi, A., et al. (2018). MMP2 and MMP9 are associated with apical periodontitis progression and might be modulated by TLR2 and MyD88. Braz. Dent. J. 29, 43-47. doi: 10.1590/0103-6440201801731

Becker, F., Gavins, F. N. E., Fontenot, J., Jordan, P., Yun, J. Y., Scott, R., et al. (2019a). Dynamic gut microbiome changes following regional intestinal lymphatic obstruction in primates. Pathophysiology 26, 253-261. doi: 10.1016/j. pathophys.2019.06.004 blood flow and their impact on the development of periodontal disease as well as support for the treatment of LGG gingival blood flow. However, the specific mechanisms and clinical effect of HFD and LGG on periodontal disease require further research.

\section{DATA AVAILABILITY STATEMENT}

The raw data supporting the conclusions of this article will be made available by the authors, without undue reservation.

\section{ETHICS STATEMENT}

The animal study was reviewed and approved by the Institutional Animal Care and Use Committee (IACUC \#19592) of the University of Louisville, KY.

\section{AUTHOR CONTRIBUTIONS}

The authors confirm that they are the original contributors of this work and all of them approved it for its publication.

\section{FUNDING}

This work was supported by NIH grants, HL139047, AR-71789, and DK116591.

\section{ACKNOWLEDGMENTS}

We thank other lab members for their continued help and support.

Becker, F., Romero, E., Goetzmann, J., Hasselschwert, D. L., Dray, B., Vanchiere, J., et al. (2019b). Endogenous specialized proresolving mediator profiles in a novel experimental model of lymphatic obstruction and intestinal inflammation in African green monkeys. Am. J. Pathol. 189, 1953-1972. doi: 10.1016/j.ajpath.2019.05.013

Blasco-Baque, V., Serino, M., Vergnes, J. N., Riant, E., Loubieres, P., Arnal, J. F., et al. (2012). High-fat diet induces periodontitis in mice through lipopolysaccharides (LPS) receptor signaling: protective action of estrogens. PLoS One 7:e48220. doi: 10.1371/journal.pone.0048220

Bolger, A. M., Lohse, M., and Usadel, B. (2014). Trimmomatic: a flexible trimmer for Illumina sequence data. Bioinformatics 30, 2114-2120. doi: 10.1093/ bioinformatics/btu170

Breivik, T., Gundersen, Y., Osmundsen, H., Fonnum, F., and Opstad, P. K. (2006). Neonatal dexamethasone and chronic tianeptine treatment inhibit ligature-induced periodontitis in adult rats. J. Periodontal Res. 41, 23-32. doi: 10.1111/j.1600-0765.2005.00833.x

Bui, C., Petrofsky, J., Berk, L., Shavlik, D., Remigio, W., and Montgomery, S. (2010). Acute effect of a single high-fat meal on forearm blood flow, blood pressure and heart rate in healthy male Asians and Caucasians: a pilot study. Southeast Asian J. Trop. Med. Public Health 41, 490-500.

Cekici, A., Kantarci, A., Hasturk, H., and Van Dyke, T. E. (2014). Inflammatory and immune pathways in the pathogenesis of periodontal disease. Periodontol. 64, 57-80. doi: 10.1111/prd.12002

Chen, X., and Li, Y. (2009). Role of matrix metalloproteinases in skeletal muscle: migration, differentiation, regeneration and fibrosis. Cell Adhes. Migr. 3, 337-341. doi: 10.4161/cam.3.4.9338 
Chen, T., Shi, Y., Wang, X., Wang, X., Meng, F., Yang, S., et al. (2017). Highthroughput sequencing analyses of oral microbial diversity in healthy people and patients with dental caries and periodontal disease. Mol. Med. Rep. 16, 127-132. doi: 10.3892/mmr.2017.6593

Chivero, E. T., Ahmad, R., Thangaraj, A., Periyasamy, P., Kumar, B., Kroeger, E., et al. (2019). Cocaine induces inflammatory gut milieu by compromising the mucosal barrier integrity and altering the gut microbiota colonization. Sci. Rep. 9:12187. doi: 10.1038/s41598-019-48428-2

Clarke, N. G., and Shephard, B. C. (1984). The effects of epinephrine and nicotine on gingival blood flow in the rabbit. Arch. Oral Biol. 29, 789-793. doi: 10.1016/0003-9969(84)90008-6

Clarke, N. G., Shephard, B. C., and Hirsch, R. S. (1981). The effects of intraarterial epinephrine and nicotine on gingival circulation. Oral Surg. Oral Med. Oral Pathol. 52, 577-582. doi: 10.1016/0030-4220(81)90071-2

Clarke, E. L., Taylor, L. J., Zhao, C., Connell, A., Lee, J. J., Fett, B., et al. (2019). Sunbeam: an extensible pipeline for analyzing metagenomic sequencing experiments. Microbiome 7:46. doi: 10.1186/s40168-019-0658-x

Clough, G. F., Kuliga, K. Z., and Chipperfield, A. J. (2017). Flow motion dynamics of microvascular blood flow and oxygenation: evidence of adaptive changes in obesity and type 2 diabetes mellitus/insulin resistance. Microcirculation 24:e12331. doi: 10.1111/micc.12331

Cueno, M. E., and Ochiai, K. (2018). Gingival periodontal disease (PD) levelbutyric acid affects the systemic blood and brain organ: insights into the systemic inflammation of periodontal disease. Front. Immunol. 9:1158. doi: 10.3389/fimmu.2018.01158

Cueno, M. E., Saito, Y., and Ochiai, K. (2016). Periodontal disease level-butyric acid amounts locally administered in the rat gingival mucosa induce ER stress in the systemic blood. Microb. Pathog. 94, 70-75. doi: 10.1016/j. micpath.2015.10.021

Curtis, M. A., Diaz, P. I., and Van Dyke, T. E. (2020). The role of the microbiota in periodontal disease. Periodontol. 83, 14-25. doi: 10.1111/prd.12296

Declèves, A. E., Rychak, J. J., Smith, D. J., and Sharma, K. (2013). Effects of high-fat diet and losartan on renal cortical blood flow using contrast ultrasound imaging. Am. J. Physiol. Renal Physiol. 305, F1343-F1351. doi: 10.1152/ajprenal.00326.2013

De Luca, F., and Shoenfeld, Y. (2019). The microbiome in autoimmune diseases. Clin. Exp. Immunol. 195, 74-85. doi: 10.1111/cei.13158

Donnini, S., Morbidelli, L., Taraboletti, G., and Ziche, M. (2004). ERK1-2 and p38 MAPK regulate MMP/TIMP balance and function in response to thrombospondin-1 fragments in the microvascular endothelium. Life Sci. 74, 2975-2985. doi: 10.1016/j.lfs.2003.09.075

Fujita, Y., and Maki, K. (2016). High-fat diet-induced obesity triggers alveolar bone loss and spontaneous periodontal disease in growing mice. BMC Obes. 3:1. doi: 10.1186/s40608-016-0082-8

Gao, L., Xu, T., Huang, G., Jiang, S., Gu, Y., and Chen, F. (2018). Oral microbiomes: more and more importance in oral cavity and whole body. Protein Cell 9, 488-500. doi: 10.1007/s13238-018-0548-1

Gatej, S. M., Marino, V., Bright, R., Fitzsimmons, T. R., Gully, N., Zilm, P., et al. (2018). Probiotic Lactobacillus rhamnosus GG prevents alveolar bone loss in a mouse model of experimental periodontitis. J. Clin. Periodontol. 45, 204-212. doi: 10.1111/jcpe.12838

George, A. K., Behera, J., Kelly, K. E., Mondal, N. K., Richardson, K. P., and Tyagi, N. (2018). Exercise mitigates alcohol induced endoplasmic reticulum stress mediated cognitive impairment through ATF6-herp signaling. Sci. Rep. 8:5158. doi: 10.1038/s41598-018-23568-z

George, A. K., Singh, M., Pushpakumar, S., Homme, R. P., Hardin, S. J., and Tyagi, S. C. (2020). Dysbiotic 1-carbon metabolism in cardiac muscle remodeling. J. Cell. Physiol. 235, 2590-2598. doi: 10.1002/jcp.29163

Graves, D. T., Corrêa, J. D., and Silva, T. A. (2019). The oral microbiota is modified by systemic diseases. J. Dent. Res. $98,148-156$. doi: 10.1177/0022 034518805739

Greiner, T., and Backhed, F. (2011). Effects of the gut microbiota on obesity and glucose homeostasis. Trends Endocrinol. Metab. 22, 117-123. doi: 10.1016/j. tem.2011.01.002

Gruner, D., Paris, S., and Schwendicke, F. (2016). Probiotics for managing caries and periodontitis: systematic review and meta-analysis. J. Dent. 48, 16-25. doi: 10.1016/j.jdent.2016.03.002

Hajishengallis, G. (2015). Periodontitis: from microbial immune subversion to systemic inflammation. Nat. Rev. Immunol. 15, 30-44. doi: 10.1038/nri3785
He, C., Cheng, D., Peng, C., Li, Y., Zhu, Y., and Lu, N. (2018). High-fat diet induces dysbiosis of gastric microbiota prior to gut microbiota in association with metabolic disorders in mice. Front. Microbiol. 9:639. doi: 10.3389/ fmicb.2018.00639

Hersoug, L. G., Møller, P., and Loft, S. (2018). Role of microbiota-derived lipopolysaccharide in adipose tissue inflammation, adipocyte size and pyroptosis during obesity. Nutr. Res. Rev. 31, 153-163. doi: 10.1017/ S0954422417000269

Huang, R., Ning, H., Shen, M., Li, J., Zhang, J., and Chen, X. (2017). Probiotics for the treatment of atopic dermatitis in children: a systematic review and meta-analysis of randomized controlled trials. Front. Cell. Infect. Microbiol. 7:392. doi: $10.3389 /$ fcimb.2017.00392

Jandhyala, S. M., Talukdar, R., Subramanyam, C., Vuyyuru, H., Sasikala, M., and Reddy, D. N. (2015). Role of the normal gut microbiota. World J. Gastroenterol. 21, 8787-8803. doi: 10.3748/wjg.v21.i29.8787

Jia, X., Jia, L., Mo, L., Yuan, S., Zheng, X., He, J., et al. (2019). Berberine ameliorates periodontal bone loss by regulating gut microbiota. J. Dent. Res. 98, 107-116. doi: 10.1177/0022034518797275

Jiang, Q., Kainulainen, V., Stamatova, I., Korpela, R., and Meurman, J. H. (2018). Lactobacillus rhamnosus GG in experimental oral biofilms exposed to different carbohydrate sources. Caries Res. 52, 220-229. doi: 10.1159/000479380

Jin, J., Zhang, X., Lu, Z., Perry, D. M., Li, Y., Russo, S. B., et al. (2013). Acid sphingomyelinase plays a key role in palmitic acid-amplified inflammatory signaling triggered by lipopolysaccharide at low concentrations in macrophages. Am. J. Physiol. Endocrinol. Metab. 305, E853-E867. doi: 10.1152/ ajpendo.00251.2013

Komaki, M. (2019). Pericytes in the periodontal ligament. Adv. Exp. Med. Biol. 1122, 169-186. doi: 10.1007/978-3-030-11093-2_10

Komazaki, R., Katagiri, S., Takahashi, H., Maekawa, S., Shiba, T., Takeuchi, Y., et al. (2017). Periodontal pathogenic bacteria, Aggregatibacter actinomycetemcomitans affect non-alcoholic fatty liver disease by altering gut microbiota and glucose metabolism. Sci. Rep. 7:13950. doi: 10.1038/ s41598-017-14260-9

Kozich, J. J., Westcott, S. L., Baxter, N. T., Highlander, S. K., and Schloss, P. D. (2013). Development of a dual-index sequencing strategy and curation pipeline for analyzing amplicon sequence data on the MiSeq Illumina sequencing platform. Appl. Environ. Microbiol. 79, 5112-5120. doi: 10.1128/ AEM.01043-13

Li, Y., Lu, Z., Zhang, X., Yu, H., Kirkwood, K. L., Lopes-Virella, M. F., et al. (2015). Metabolic syndrome exacerbates inflammation and bone loss in periodontitis. J. Dent. Res. 94, 362-370. doi: 10.1177/0022034514561658

Lin, P. W., Myers, L. E., Ray, L., Song, S. -C., Nasr, T. R., Berardinelli, A. J., et al. (2009). Lactobacillus rhamnosus blocks inflammatory signaling in vivo via reactive oxygen species generation. Free Radic. Biol. Med. 47, 1205-1211. doi: $10.1016 /$ j.freeradbiomed.2009.07.033

Livak, K. J., and Schmittgen, T. D. (2001). Analysis of relative gene expression data using real-time quantitative PCR and the 2(-Delta Delta C(T)) method. Methods 25, 402-408. doi: 10.1006/meth.2001.1262

Lozupone, C. A., Stombaugh, J. I., Gordon, J. I., Jansson, J. K., and Knight, R. (2012). Diversity, stability and resilience of the human gut microbiota. Nature 489, 220-230. doi: 10.1038/nature11550

Lu, Y., Fan, C., Liang, A., Fan, X., Wang, R., Li, P., et al. (2018). Effects of SCFA on the DNA methylation pattern of adiponectin and resistin in highfat-diet-induced obese male mice. Br. J. Nutr. 120, 385-392. doi: 10.1017/ S0007114518001526

Lu, R., Meng, H., Gao, X., Xu, L., and Feng, X. (2014). Effect of non-surgical periodontal treatment on short chain fatty acid levels in gingival crevicular fluid of patients with generalized aggressive periodontitis. J. Periodontal Res. 49, 574-583. doi: $10.1111 /$ jre.12137

Mariat, D., Firmesse, O., Levenez, F., Guimarăes, V., Sokol, H., Doré, J., et al. (2009). The Firmicutes/Bacteroidetes ratio of the human microbiota changes with age. BMC Microbiol. 9:123. doi: 10.1186/1471-2180-9-123

Mitra, R., Qiao, J., Madhavan, S., O’Neil, G. L., Ritchie, B., Kulkarni, P., et al. (2018). The comparative effects of high fat diet or disturbed blood flow on glycocalyx integrity and vascular inflammation. Transl. Med. Commun. 3:10. doi: 10.1186/s41231-018-0029-9

Muluke, M., Gold, T., Kiefhaber, K., Al-Sahli, A., Celenti, R., Jiang, H., et al. (2016). Diet-induced obesity and its differential impact on periodontal bone loss. J. Dent. Res. 95, 223-229. doi: 10.1177/0022034515609882 
Münch, N. S., Fang, H. Y., Ingermann, J., Maurer, H. C., Anand, A., Kellner, V., et al. (2019). High-fat diet accelerates carcinogenesis in a mouse model of Barrett's esophagus via interleukin 8 and alterations to the gut microbiome. Gastroenterology 157, 492.e2-506.e2. doi: 10.1053/j.gastro. 2019.04.013

Murphy, E. A., Velazquez, K. T., and Herbert, K. M. (2015). Influence of high-fat diet on gut microbiota: a driving force for chronic disease risk. Curr. Opin. Clin. Nutr. Metab. Care 18, 515-520. doi: 10.1097/ MCO.0000000000000209

Ng, M., Fleming, T., Robinson, M., Thomson, B., Graetz, N., Margono, C., et al. (2014). Global, regional, and national prevalence of overweight and obesity in children and adults during 1980e2013: a systematic analysis for the global burden of disease study 2013. Lancet 384, 766-781. doi: 10.1016/ S0140-6736(14)60460-8

Nguyen, C. M., Kim, J. W., Quan, V. H., Nguyen, B. H., and Tran, S. D. (2015). Periodontal associations in cardiovascular diseases: the latest evidence and understanding. J. Oral Biol. Craniofac. Res. 5, 203-206. doi: 10.1016/j. jobcr.2015.06.008

Niederman, R., Buyle-Bodin, Y., Lu, B. Y., Robinson, P., and Naleway, C. (1997). Short-chain carboxylic acid concentration in human gingival crevicular fluid. J. Dent. Res. 76, 575-579. doi: 10.1177/00220345970760010801

Ohsugi, Y., Nagashima, Y., Nakatsu, S., Sato, K., Chiba, A., Fujinaka, H., et al. (2019). Age-related changes in gingival blood flow parameters measured using laser speckle flowmetry. Microvasc. Res. 122, 6-12. doi: 10.1016/j. mvr.2018.10.010

Pacios, S., Kang, J., Galicia, J., Gluck, K., Patel, H., Ovaydi-Mandel, A., et al. (2012). Diabetes aggravates periodontitis by limiting repair through enhanced inflammation. FASEB J. 26, 1423-1430. doi: 10.1096/ f. 11-196279

Payne, M. A., Hashim, A., Alsam, A., Joseph, S., Aduse-Opoku, J., Wade, W. G., et al. (2019). Horizontal and vertical transfer of oral microbial dysbiosis and periodontal disease. J. Dent. Res. 98, 1503-1510. doi: $10.1177 / 0022034519877150$

Potter, G. D., Cade, J. E., Grant, P. J., and Hardie, L. J. (2016). Nutrition and the circadian system. Br. J. Nutr. 116, 434-442. doi: 10.1017/ S0007114516002117

Qiqiang, L., Huanxin, M., and Xuejun, G. (2012). Longitudinal study of volatile fatty acids in the gingival crevicular fluid of patients with periodontitis before and after nonsurgical therapy. J. Periodontal Res. 47, 740-749. doi: 10.1111/j.1600-0765.2012.01489.x

Rodrigues, S. F., and Granger, D. N. (2015). Blood cells and endothelial barrier function. Tissue Barriers 3:e978720. doi: 10.4161/21688370.2014.978720

Sanchez, M., Darimont, C., Drapeau, V., Emady-Azar, S., Lepage, M., Rezzonico, E., et al. (2014). Effect of Lactobacillus rhamnosus CGMCC1.3724 supplementation on weight loss and maintenance in obese men and women. Br. J. Nutr. 111, 1507-1519. doi: 10.1017/S0007114513003875

Scheithauer, T. P., Dallinga-Thie, G. M., de Vos, W. M., Nieuwdorp, M., and van Raalte, D. H. (2016). Causality of small and large intestinal microbiota in weight regulation and insulin resistance. Mol. Metab. 5, 759-770. doi: 10.1016/j.molmet.2016.06.002

Scott, K. P., Gratz, S. W., Sheridan, P. O., Flint, H. J., and Duncan, S. H. (2013). The influence of diet on the gut microbiota. Pharmacol. Res. 69, 52-60. doi: 10.1016/j.phrs.2012.10.020

Semenkovich, C. F., Danska, J., Darsow, T., Dunne, J. L., Huttenhower, C., Insel, R. A., et al. (2015). American diabetes association and JDRF research symposium: diabetes and the microbiome. Diabetes 64, 3967-3977. doi: $10.2337 / \mathrm{db} 15-0597$

Semenoff, T. A., Semenoff-Segundo, A., Bosco, A. F., Nagata, M. J., Garcia, V. G., and Biasoli, E. R. (2008). Histometric analysis of ligature-induced periodontitis in rats: a comparison of histological section planes. J. Appl. Oral Sci. 16, 251-256. doi: 10.1590/s1678-77572008000400005

Shimizu, M., Hashiguchi, M., Shiga, T., Tamura, H. O., and Mochizuki, M. (2015). Meta-analysis: effects of probiotic supplementation on lipid profiles in normal to mildly hypercholesterolemic individuals. PLoS One 10:e0139795. doi: 10.1371/journal.pone.0139795

Soolari, A. S., Champagne, C., Punzi, J. S., Amar, S., and Van Dyke, T. E. (1999). Serum modulation of neutrophil response to Porphyromonas gingivalis LPS in periodontal disease. J. Int. Acad. Periodontol. 1, 101-109.
Stanisic, D., George, A. K., Smolenkova, I., Singh, M., and Tyagi, S. C. (2020). Hyperhomocysteinemia: an instigating factor for periodontal disease. Can. J. Physiol. Pharmacol. 99, 115-123. doi: 10.1139/cjpp-2020-0224

Stanisic, D., Obradovic, O., Vujovic, S., Jovanovic, M., and Zivkovic, V. (2019). Connection of periodontal disease and diabetes mellitus: the role of matrix metalloproteinases and oxidative stress. Serbian J. Exp. Clin. Res. doi: 10.2478/ sjecr-2019-0051

Susin, C., and Rösing, C. K. (2003). Effect of variable moderate chronic stress on ligature-induced periodontal disease in Wistar rats. Acta Odontol. Scand. 61, 273-277. doi: 10.1080/00016350310005574

Takigawa, S., Sugano, N., Nishihara, R., Koshi, R., Murai, M., Yoshinuma, N., et al. (2008). The effect of butyric acid on adhesion molecule expression by human gingival epithelial cells. J. Periodontal Res. 43, 386-390. doi: 10.1111/j.1600-0765.2007.01048.x

Tolhurst, G., Heffron, H., Lam, Y. S., Habib, A. M., Diakogiannaki, E., Cameron, J., et al. (2012). Short-chain fatty acids stimulate glucagon-like peptide-1 secretion via the G-protein-coupled receptor FFAR2. Diabetes 61, 364-371. doi: 10.2337/ db11-1019

Torres, J., Mehandru, S., Colombel, J. F., and Peyrin-Biroulet, L. (2017). Crohn's disease. Lancet 389, 1741-1755. doi: 10.1016/S0140-6736(16)31711-1

Tremaroli, V., and Bäckhed, F. (2012). Functional interactions between the gut microbiota and host metabolism. Nature 489, 242-249. doi: 10.1038/ nature 11552

Tsuda, H., Ochiai, K., Suzuki, N., and Otsuka, K. (2010). Butyrate, a bacterial metabolite, induces apoptosis and autophagic cell death in gingival epithelial cells. J. Periodontal Res. 45, 626-634. doi: 10.1111/j.1600-0765.2010.01277.x

Turnbaugh, P. J., Backhed, F., Fulton, L., and Gordon, J. I. (2008). Diet-induced obesity is linked to marked but reversible alterations in the mouse distal gut microbiome. Cell Host Microbe 3, 213-223. doi: 10.1016/j.chom.2008.02.015

Turnbaugh, P. J., Ley, R. E., Hamady, M., Fraser-Liggett, C., Knight, R., and Gordon, J. I. (2007). The human microbiome project: exploring the microbial part of ourselves in a changing world. Nature 449, 804-810. doi: 10.1038/ nature 06244

Veronesi, F., Borsari, V., Sartori, M., Orciani, M., Mattioli-Belmonte, M., and Fini, M. (2018). The use of cell conditioned medium for musculoskeletal tissue regeneration. J. Cell. Physiol. 233, 4423-4442. doi: 10.1002/jcp.26291

Virto, L., Cano, P., Jiménez-Ortega, V., Fernández-Mateos, P., González, J., Esquifino, A. I., et al. (2018a). Obesity and periodontitis: an experimental study to evaluate periodontal and systemic effects of comorbidity. J. Periodontol. 89, 176-185. doi: 10.1902/jop.2017.170355

Virto, L., Haugen, H. J., Fernández-Mateos, P., Cano, P., González, J., Jiménez-Ortega, V., et al. (2018b). Melatonin expression in periodontitis and obesity: an experimental in-vivo investigation. J. Periodontal Res. 53, 825-831. doi: 10.1111/jre.12571

Wei, L., Yue, F., Xing, L., Wu, S., Shi, Y., Li, J., et al. (2020). Constant light exposure alters gut microbiota and promotes the progression of Steatohepatitis in high fat diet rats. Front. Microbiol. 11:1975. doi: 10.3389/ fmicb.2020.01975

Wilde, D. W., Massey, K. D., Walker, G. K., Vollmer, A., and Grekin, R. J. (2000). High-fat diet elevates blood pressure and cerebrovascular muscle $\mathrm{Ca}^{2+}$ current. Hypertension 35, 832-837. doi: 10.1161/01.hyp.35.3.832

Wohlfeil, M., Scharf, S., Siegelin, Y., Schacher, B., Oremek, G. M., Sauer-Eppel, H., et al. (2012). Increased systemic elastase and C-reactive protein in aggressive periodontitis (CLOI-D-00160R2). Clin. Oral Investig. 16, 1199-1207. doi: 10.1007/s00784-011-0627-7

Wood, D. E., Lu, J., and Langmead, B. (2019). Improved metagenomic analysis with kraken 2. Genome Biol. 20:257. doi: 10.1186/s13059-019-1891-0

Yang, Y., Zhang, Y., Xu, Y., Luo, T., Ge, Y., Jiang, Y., et al. (2019). Dietary methionine restriction improves the gut microbiota and reduces intestinal permeability and inflammation in high-fat-fed mice. Food Funct. 10, 5952-5968. doi: $10.1039 / \mathrm{c} 9$ fo00766k

Yu, X. L., Chan, Y., Zhuang, L., Lai, H. C., Lang, N. P., Keung Leung, W., et al. (2019). Intra-oral single-site comparisons of periodontal and periimplant microbiota in health and disease. Clin. Oral Implants Res. 30, 760-776. doi: 10.1111/clr.13459

Yuan, W., Ma, C., Zhou, Y., Wang, M., Zeng, G., and Huang, Q. (2019). Negative regulation of eNOS-NO signaling by over-SUMOylation of PPAR $\gamma$ contributes to insulin resistance and dysfunction of vascular endothelium in rats. Vasc. Pharmacol. 122-123:106597. doi: 10.1016/j.vph.2019.106597 
Yucel, O. O., Berker, E., Mesci, L., Eratalay, K., Tepe, E., and Tezcan, İ. (2015). Analysis of TNF- $\alpha$ (-308) polymorphism and gingival crevicular fluid TNF- $\alpha$ levels in aggressive and chronic periodontitis: a preliminary report. Cytokine 72, 173-177. doi: 10.1016/j.cyto.2015.01.001

Zhang, M., and Yang, X. J. (2016). Effects of a high fat diet on intestinal microbiota and gastrointestinal diseases. World J. Gastroenterol. 22, 8905-8909. doi: 10.3748 /wjg.v22.i40.8905

Zhao, G., Nyman, M., and Jönsson, J. A. (2006). Rapid determination of shortchain fatty acids in colonic contents and faeces of humans and rats by acidified water-extraction and direct-injection gas chromatography. Biomed. Chromatogr. 20, 674-682. doi: 10.1002/bmc.580
Conflict of Interest: The authors declare that the research was conducted in the absence of any commercial or financial relationships that could be construed as a potential conflict of interest.

Copyright (c) 2021 Stanisic, Jeremic, Majumder, Pushpakumar, George, Singh and Tyagi. This is an open-access article distributed under the terms of the Creative Commons Attribution License (CC BY). The use, distribution or reproduction in other forums is permitted, provided the original author(s) and the copyright owner(s) are credited and that the original publication in this journal is cited, in accordance with accepted academic practice. No use, distribution or reproduction is permitted which does not comply with these terms. 